\title{
The Use of Multimedia Learning Resource Sharing (MLRS) in Developing Sharing Knowledge at Schools
}

\author{
Munir \\ Department of Computer Science, Indonesia University of Education \\ munir@upi.edu
}

\begin{abstract}
This study aimed to develop school community to share knowledge by using Multimedia Learning Resource Sharing (MLRS). MLRS was developed as a means for educators and learners across schools to share online based internet. To measure MLRS's acceptance as a medium of sharing knowledge, Technology Acceptance Model (TAM) was used as a model of approach. This study tried to explore factors that were dominant, interrelated and influential to the level of MLRS's acceptance in sharing knowledge. Structural Equation Modeling (SEM) was used to analyze the data. The present study revealed that user Content Management (CM) affected Perceived Ease of Use (PEoU), while Content management (CM) and PEoU affected the benefits of Perceived Usefulness (PU). PEoU and PU affected Intention to Use (ITU); and ITU affected Actual System Usage (ASU). In general, the presence of MLRS received a positive acceptance from users in developing sharing knowledge between Senior High schools in West Java.
\end{abstract}

Keywords: Multimedia Learning Resource Sharing (MLRS); Sharing Knowledge Community

\section{Introduction}

The rapid growth of Internet among society is an opportunity that needs to be followed up by an optimal Internet empowerment. Educational institution is one of the parties interested in making Internet as a medium of modern learning. In this context, Multimedia Learning is an option that might be chosen by an educational institution in a computerbased modern learning. The availability of computer facilities and internet technology is the opportunity to create modern learning and sharing knowledge community. One of the challenges for educational institutions in the era of information technology nowadays is to provide learning for each student at the right time without being bordered by the location where he/she resides. Concerning this borderless learning, "The ability to learn faster than your competitors maybe the only sustainable competitive advantage"[1]. One solution to address these challenges is to facilitate independent learning. Independent study has rapidly developed in accordance with the development and convergence occuring in information technology and telecommunication. Various technologies and applications have been created in order to support the operational activities of human life and institutions, including teaching and learning activities such as Multimedia Learning.

Student performance in teaching and learning process can be seen through their interactions in Multimedia Learning. Electronic communication is developed through a combination of learning content, teaching and learning opportunity.

MLRS is trying to be one solution in changing the mindset and culture of learners towards the use of social networks into sharing knowledge community. By observing the Internet users in Indonesia, we could observe that teenagers tend to have a great interest in the development of web-based Internet application 2.0, i.e. the Internet application technology that forwards communication and social networking. 
The presence of social networks gets overwhelming responses by teenagers, who are mostly students. Since Multimedia Learning is still a new experience for most students and teachers, it has been indicated that it will be very useful when it is used in the students' learning. Students are advised to correlate cyber learning with their learning experiences in the class. The goal is to make students understand the different forms of Multimedia Learning with the assumption that they will personally be a developer of Cyber Learning in the future.

In accordance with the above discussion, MLRS is an option to encourage the creation of cyber-learning to promote e-literacy, sharing knowledge and the creation of an effective modern learning.

Learning model such as Multimedia Learning Resource Sharing (MLRS) was developed in cooperation among Indonesia University of Education (UPI) and the Department of Education and Culture of West Java. The presence of MLRS has not been widely known especially at the level of effectiveness and user acceptance. The present study was conducted to determine what factors influenced the use of MLRS in developing sharing knowledge between schools in West Java.

Based on the above explanation, then the problem of this study is formulated as follows: What factors are affecting the use of Multimedia Learning Resource Sharing (MLRS) in developing Sharing Knowledge?

\section{A Brief Review of Related Researches and Works}

\subsection{The Initial Model of MLRS}

Multimedia Learning Resource Sharing (MLRS) is a school system facility for a wide range of science. Through MLRS, the process of knowledge development not only happens in the classroom where educators provide lessons centrally in the same direction, but also with the help of computer equipment and networks that learners can actively participate in the process of teaching and learning outside the classroom [2-3].

According to the data content, information and knowledge, MLRS is one form of Knowledge Management (KM), but the process is different [7-9].The activity of knowledge centered on the process of individual psychology such as experiences, perceptions, emotions and motivations [10-12]. Within the organization, there are four main activities of knowledge, namely (1) knowledge acquisition, refers to the internalization of information, (2) knowledge creation, which is associated with the creation of new knowledge (3) knowledge distribution, which is the distribution phase of knowledge within the organization, (4) knowledge application, which refers to the practical use of knowledge.

In the perspective of the educational environment of academic knowledge, learning community should begin at the individual stage, by building a department that manages knowledge in organizations, creating an interesting domain across departments, creating a knowledge network within organizations as well as other organizations. Thus, in the context of academic knowledge, the strategies used are individual strategy, institutional strategy and network strategy.

Knowledge is built through sharing within the community, from individuals through a team and a group to the organizational level.

\subsection{Technology Acceptance Model (TAM)}

As a system, we need to know the level of effectiveness and efficiency of MLRS. Several models have been constructed to analyze and understand the factors affecting the use of MLRS. One of indicators that can be used is the Technology Acceptance Model (TAM) which was developed by Davis [11]. 
TAM model explains the behavior of the users of ICT, which is based on trust (belief), attitude, intention, and user behavior relationship. TAM model collocates the attitude factor of each user's behavior with the two variables namely usefulness and ease of use that will explain user behaviors [11]. TAM model has five constructs namely Perceived Ease of Use, Perceived Usefulness, Attitude towards Use, Behavioral Intention to Use, and Actual System Usage [11]. Perceived Ease of Use is user perceptions about ease of use of a technology as a measure of credential. Ease of Use indicators of MLRS's usages are: (i) easy to learn, (ii) easy to do certain activities needed by users, (iii) easy to increase user skills, and (iv) easy to operate.

Perceived Usefulness is user perception on the usefulness as a measure that MLRS is trustworthy and beneficial to those who use it. The usefulness of MLRS is: (i) encompassing the dimensions of learning in terms of: easier to use, beneficial, more productive, and (ii) effective in terms of: increasing effectiveness, and developing learning performance. Attitude toward Use is user attitude toward MLRS's usage in terms of acceptance and refusal as an impact of someone using MLRS. Behavioral Intention to Use is user attitude to remain using MLRS. The level of MLRS's usage by learners can be predicted from the attitude of concern for MLRS.

On the other occasion, Fishbein, M., \& Ajzen, I. [16] conducted a study with TAM as a model for analyzing the behavior of the use of e-learning by students at some colleges. In that study, the external variables that affected the user's comprehension on the usefulness and ease of e-learning were classified into individual factors, social factors and organizational factors. Another study by Hong, W., Tong, J., Wong, W., and Tam, K. [18] applied TAM to the digital library, in which they found the influence of two external variables i.e. individual differences and system characteristics to the ease of use, as well as the influence of system characteristics on perceptions of usefulness. Individual differences consist of Internet self-efficacy or ISE and knowledge of search domain. System characteristics used were the characteristics of the studies on retrieval data systems, i.e. the digital library.

\section{Structural Equation Model (SEM)}

The methodology used in this study was the explanatory method of causal relation from the observed and studied variables. Randomized sampling was taken from educators and learners of Senior High School in West Java, through cooperation between Indonesia University of Education and the Department of Education and Culture of West Java Indonesia.

Data were collected by using a survey method through questionnaire administered to 159 educators and learners. Descriptive statistical analysis was performed to examine the size of frequency distribution of data centralization and dispersion of the characteristics of the sample and the indicators which include exogenous and endogenous constructs.

Exogenous constructs included Portal Design and Interface (PDI) with the indicators: functionality (X1), Interface (X2) and Navigator (X3), and Content Management (CM), which consisted of cognition (X4), Skill (X5), and affection (X6). Learner Motivation (LM) is consisting of accessibility (X7), visibility (X8), and curiosity (X9). Environment Influence (EI) consists of policy(X10), facility (X11); while endogenous includes Perceived Ease of Use (PEoU) with the indicators: comprehensible (Y1), user friendly (Y2) and easy to use (Y3). Perceived Usefulness (PU) consists of indicators: faster (Y4), performance improved (Y5), more efficient (Y6), easier (Y7), increased productivity (Y8) and effectiveness (Y9). Behavioral Intention to Use (ITU) consists of: an intention to use (Y10) and an intention to share (Y11). Actual System Usage (ASU) consists of: the frequency of the use (Y12) and the use of a longer period of time (Y13) from the MLRS.

A Likert scale was used to score each item question. Structural Equation Modeling (SEM) was used to analyze the data to determine the percentage level of the relationship 
and influence between variables in a path diagram. Processing and analyzing data were conducted by multivariate techniques of Structural Equation Model.

From the nine constructs proposed, structural equation was obtained to form the research model:

$$
\begin{aligned}
& \mathrm{PEoU}=\gamma 11 \mathrm{PDI}+\gamma 12 \mathrm{CM}+\gamma 13 \mathrm{LM}+\zeta 1 \ldots(1) \\
& \mathrm{PU}=\gamma 24 \mathrm{CM}+\gamma 24 \mathrm{EI}+\beta 21 \mathrm{PEoU}+\zeta 2 \ldots(2) \\
& \mathrm{ITU}=\beta 46 \mathrm{PEoU}+\beta 34 \mathrm{PU}++\zeta 3 \ldots(3) \\
& \mathrm{ASU}=\beta 56 \mathrm{ITU}+\zeta 4 \ldots(4)
\end{aligned}
$$

\section{Experiments and Analysis}

Data from 159 respondents were collected and analyzed statistically. Based on the reference values from $r$ table (values of $r$ product moment), it was noted that the number of sample $(\mathrm{N})=159$, and the significant level $(\alpha) 0.01(1 \%)=0.235$ and $(\alpha) 0.05(5 \%)=$ 0.167 and it was stated that the item instrument was invalid. According to the Reliability Test, the data were considered reliable if the value of CA (Cronbach's Alpha) was between $0.60-0.70[19]$. The coefficient of Cronbach's Alpha reliability above is 0.817 . According to the criteria, this value is greater than 0.60 , and thus the data derived from the questionnaire had a good level of reliability.

\subsection{Testing Model}

The testing model proposed earlier by the author can be seen in Figure 1 below:

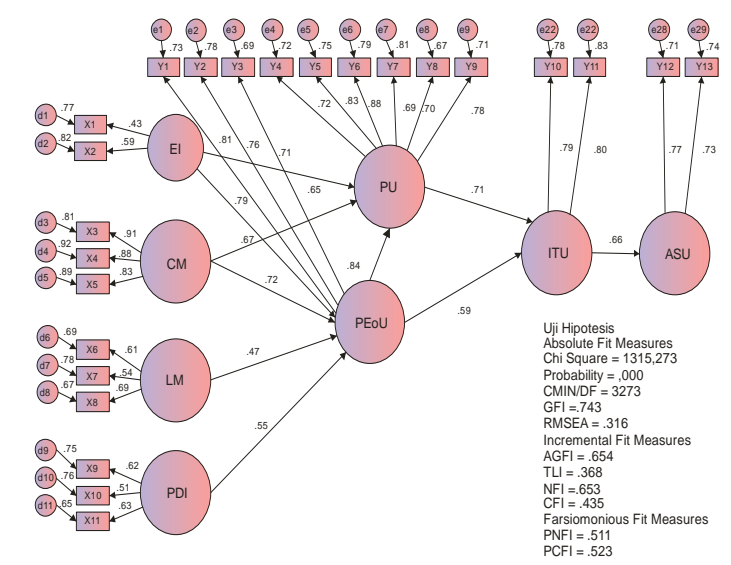

Figure 1. The Initial Model of MLRS's Usage

The probability level revealed in the picture above is $(\mathrm{P})=0000$. It is not in accordance with the recommended value, i.e. the probability $(\mathrm{P}) \geq 0.05$. Likewise with the critical value of Chi-square $\mathrm{X} 2(\mathrm{CMIN})=1315.273$ which is too large and Chi-square X2 Relative $(\mathrm{CMIN} / \mathrm{DF})=3.273>3.0, \mathrm{GFI}=0.743<0.90, \mathrm{RMSEA}=0.316>0.10, \mathrm{AGFI}=0.654<$ $0.90, \mathrm{TLI}=0.368<0.95, \mathrm{NFI}=0.653<0.90, \mathrm{CFI}=0.435<0.95, \mathrm{PNFI}=0.511<0.60$, and PCFI $=0.523<0.60$. Thus, we can modify the existing model until the model can be stated as fit.

In this study, modifications were conducted to change the parameter (index) of each possible variable. Index modification (Modification Indices) gives a decrease or reduction in Chi-square value if it was estimated. The modification was based on the value of its covariances conducted by connecting the error factor of a particular variable with the error indicator of other factors, which means that both relationships of error factors affected each other significantly. The results of the final modifications in this study can be seen in Figure 2 as follows: 


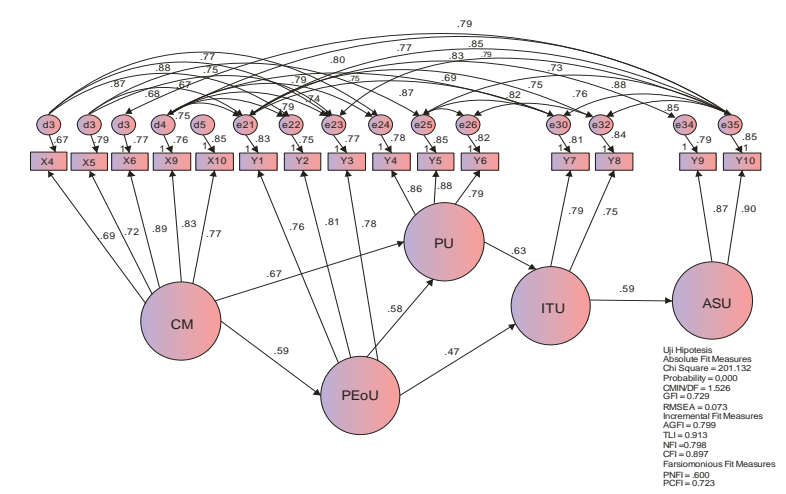

Figure 2. The Final Model of MLRS's Usage

\subsection{Hypothesis Testing}

By considering the results of the modification of existing models, there are relationships between E-resources Organization (ErO) with Perceived Usefulness (PU); Individual Differences (ID) and Perceived Ease of Use (PEoU), Perceived Ease of Use (PEoU) and Perceived Usefulness (PU), Perceived Ease of Use (PEoU) with Behavioral Intention to Use (ITU); Perceived Usefulness (PU) with Behavioral Intention to Use (ITU), and between Behavioral Intention to Use (ITU) with Actual System Usage (ASU).

The results of hypothesis testing were taken from the output results in the Estimates $\rightarrow$ Scalars $\rightarrow$ Regression Weights, as shown in Table 1:

Table 1. The Result of Hypothesis Testing of MLRS

\begin{tabular}{|l|c|l|}
\hline \multicolumn{1}{|c|}{ Hypothesis } & Sig $<\mathbf{0 . 0 5}$ & Hypothesis Result \\
\hline $\mathrm{H}_{1}(\mathrm{CM} \rightarrow$ PEoU $)$ & 0.000 & Declining $\mathrm{H}_{01}$ \\
\hline $\mathrm{H}_{2}(\mathrm{CM} \rightarrow$ PU $)$ & 0.000 & Declining $\mathrm{H}_{02}$ \\
\hline $\mathrm{H}_{3}(\mathrm{PEoU} \rightarrow$ PU $)$ & 0.000 & Declining $\mathrm{H}_{03}$ \\
\hline $\mathrm{H}_{4}(\mathrm{PEoU} \rightarrow$ ITU $)$ & 0.000 & Declining $\mathrm{H}_{04}$ \\
\hline $\mathrm{H}_{5}(\mathrm{PU} \rightarrow$ ITU $)$ & 0.000 & Declining $\mathrm{H}_{05}$ \\
\hline $\mathrm{H}_{6}(\mathrm{ITU} \rightarrow \mathrm{ASU})$ & 0.000 & Declining $\mathrm{H}_{06}$ \\
\hline
\end{tabular}

\subsection{Model Interpretation}

Based on a modification of the model and the results of hypothesis testing in this study, it is known that the use of Multimedia Learning Resource Sharing (MLRS) in developing sharing knowledge in the schools in West Java was influenced by the presence of Content Management (CM), Perceived Ease of Use (PEoU), Perceived Usefulness (PU), Behavioral Intention To Use (ITU) and Actual System Usage (ASU). The final model interpretation of the study is as follows:

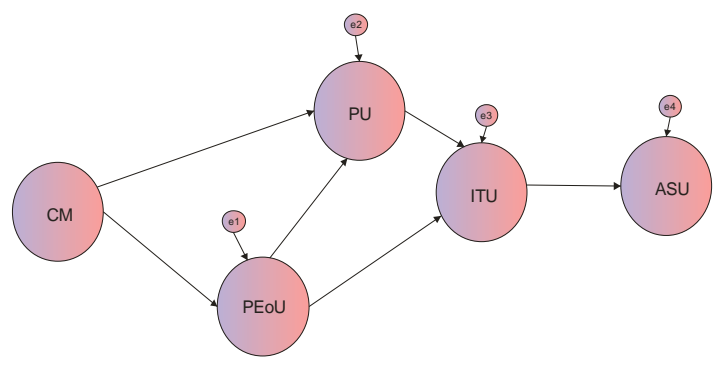

Figure 3. The Final Model Interpretation of MLRS's Usage 


\section{Discussion}

MLRS was developed based on analysis of the result on user necessity. Technology helped users to transform knowledge through browser by which the accessibility was managed in the provided database. Model of MLRS which was developed can be seen below.

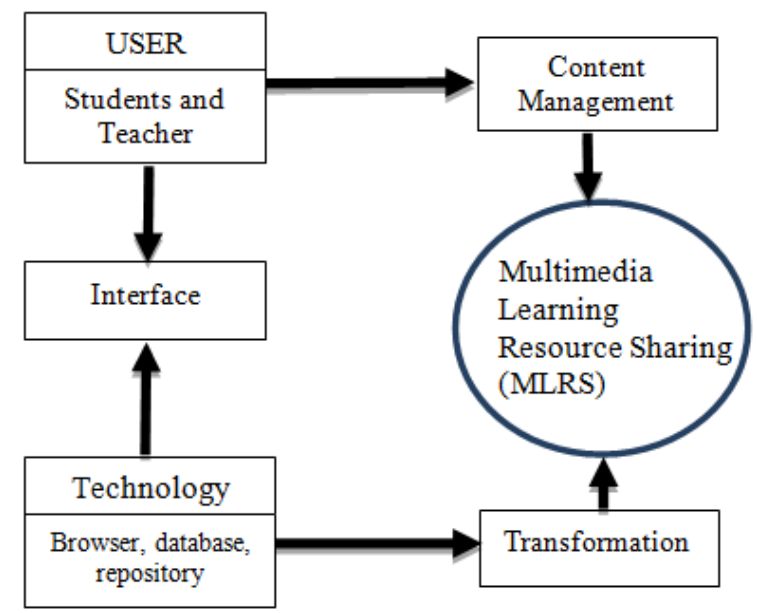

Figure 4. Model of MLRS

Model of MLRS was developed by considering the following things: (i) content management (ii) experience management, and (iii) process management. The features of the developed MLRS are (i) web-based MLRS, (ii) user authentication, (iii) upload and download facility, (iv) adjusted facility, (v) the document storage system, (vi) searching facility.

The level of user acceptance of MLRS based on statistical analysis result which consists of model testing, hypothesis testing and model interpretation showed positive results. The early model of development to test the level of MLRS acceptance consists of Exogenous constructs whose indicators include: Portal Design and Interface (PDI), Content Management (CM), Learner Motivation (LM), Environment Influence (EI), while Endogenous constructs include Perceived Ease of Use (PEoU), Perceived Usefulness (PU), Attitude Toward Using (ATU), Behavioral Intention To Use (ITU) and Actual System Usage (ASU). Apparently those indicators statistically did not cause a fit criterion of the model (see Figure 1). Therefore, it should be re-examined by conducting modification between latent variables and indicators so statistically it will become a model with fit criterion (see Figure 2).

Modifications were conducted into structural equation between latent variables i.e. $\mathrm{PEoU}=\gamma 11 \mathrm{PDI}+\gamma 12 \mathrm{CM}+\gamma 13 \mathrm{LM}+\zeta 1 \ldots(1)$ which was changed into $\mathrm{PEoU}=\gamma 13 \mathrm{CM}+\zeta 1 \ldots(2), \quad$ and $\mathrm{PU}=\gamma 46 \mathrm{CM}+\beta 34 \mathrm{PEoU}+\zeta 4 \ldots$ (3) then into $\mathrm{ITU}=\beta 32 \mathrm{PEoU}+\beta 34 \mathrm{PU}+\zeta 4 \ldots . .(4)$, and then into $\mathrm{ASU}=+\beta 34 \mathrm{ITU}+\zeta 4 \ldots . .(5) \mathrm{The}$ first and the forth structural equation changes were caused by the deletion of latent variables PDI, EI and LM into PEoU, as can be seen in the forth structural equation by forming a line influenced by CM into PeoU and PU which had the same level of influence. In addition to the changes occurred in the structural equation of $\mathrm{CM}$ which had the same level with PEoU, PU, ITU and ASU, there were also LM indicator (X9) and EI indicator (X10) which were dominant into $\mathrm{CM}$.

The results of hypothesis testing conducted were as follow: H1 (CM - PEOU) resulted in significant value (0.000), H2 (CM - PU) with significant value (0.000), H3 (PEoU PU) with significant value (0.000), H4 (PeoU -ITU) with significant value (0.000), H5 
(PU -ITU) with significant value (0.000), and H6 (ITU -ASU) with significant value $(0.000)$. Since the significant value is $(.000)<$ the significant value 0.05 , then all of them were rejected. ITU in the end affected on ASU. Since MLRS used web application, and it was well-known by students and teachers, they frequently used it through browser such as Internet Explorer on website: MLRS.org or komunitassekolah.upi.edu. According to students and teachers perceptions, MLRS was comprehensible, easy to operate and easy to use so it was easier to share knowledge and to find learning materials needed by them-without installing a specific application.

Viewed from its usefulness, by using MLRS, PU influences ITU. It means that MLRS had a faster usefulness, enhanced productivity, more effective and easier, improving performance and efficient. These created perception and desire to use MLRS. The acceptance from students and teachers reflected that MLRS was well accepted. They could experience some improvement in the terms of cognitive affective and psychomotor. Students and teachers behavior (ASU) toward the usefulness of MLRS showed a positive direction so that the desire and the interest of users were getting complex. The indicators would affect students' and teachers' actualization in sharing knowledge by using MLRS. Thus, ASU was affected by ITU and user behavior in using MLRS emerged from the desire to use it. In this study, the positive behavior in using MLRS was the frequency and the length of time.

As for the result of statistical testing showed that the dominant interrelated factors which influenced the level of MLRS acceptance were: content management (CM) which affected perception on Perceived Ease of Use (PEoU) and Perceived Usefulness (PU). Perceived Ease of Use (PEoU) affected Perceived Usefulness (PU), Perceived Ease of Use (PEoU) and Perceived Usefulness (PU) affected Behavioral Intention to Use (ITU), and Behavioral Intention to Use (ITU) affected Actual System Usage (ASU).

\section{Conclusion}

Multimedia Learning Resource Sharing (MLRS) fulfilled the criterias of developing sharing knowledge based on the result of the modified model. The factors which are dominant, correlated and influential to the level of acceptance of Multimedia Learning Resource Sharing technology are: Learner Motivation (LM)) which affects Perceived Ease of Use (PEoU), Content Management (CM) and Perceived Ease of Use (PEoU) which affect Perceived Usefulness (PU), Perceived Ease of Use (PEoU) and Perceived Usefulness (PU) which affect Behavioral Intention To Use (ITU), and Behavioral Intention To Use (ITU) which affects Actual System Usage (ASU).The usage of Multimedia Learning Resource Sharing(MLRS) in developing sharing knowledge at schools in West Java is influenced by Learner Motivation (LM)), Content Management (CM), Perceived Ease of Use (PEoU), Perceived Usefulness (PU), Behavioral Intention to Use (ITU) and Actual System Usage (ASU). In the following work, hopefully MLRS will be continuously applied and developed.

\section{References}

[1] Senge P. M., "The Fifth Discipline: The Art and Practice of the Learning Organization Currency Doubleday", New York, (1990).

[2] Davies D., "Creative learning environments in education-A systematic literature review", Thinking Skills and Creativity, [www.elsevier.com/local/tsc, vol. 8, (2013), pp. 80-91.

[3] Annetta L. A., "Investigating the impact of video games on high school students engagement and learning about genetics", Computer \& Education, [www.elsevier.com/local/compedu], vol. 53, (2009), pp. 74-85.

[4] Niyato D. and Hossain E., "Dynamics of network selection in heterogeneous wirless network: An evolutionari game approach", IEEE Transactions on Vehicular Technology, vol. 58, no. 4, (2009).

[5] Wu B., Wang A. I., Ruud A. D. And Zhang W. Z., "Extending Google Android's Application as an Educational Tool", IEEE International Confrence on Digital Game and Intelligent Toy Enhanced Learning, (2010). 
[6] Liu C. C., Cheng Y. B. and Huang C. W., "The effect of simulation games on the learning of computational problem solving", Computer \& Education, [www.elsevier.com/local/compedu], vol. 57, (2011), pp. 1907-1918.

[7] Romirez C. G. R., Almonte J. B. Tugade R. R. and Atienza R. O., "Implementation of a digital gamebase learning environment for elementry education", 2nd International Confrence on Education Technology and Computer (ICETC), (2010).

[8] Ibanez B. C., Boundier V. and Labat J. M., "Knowledge Management Approach to support a serious game development", Ninth IEEE International Confrence on Advanced Learning Technologies, (2009).

[9] Wickramasinghe N., "Knowledge-Based Enterprise: Theories and Fundamentals", Idea Group publishing, London, (2006).

[10] Jong B. S., Lai C. H., Hsia Y. T., Lin T. W. and Lu C. Y., "Using game-based cooperative learning to improve learning motivation: A studi of online game use in a operating system course", IEEE Transactions on Education, vol. 56, no. 2, (2013).

[11] Habgood M. P. J. and Ainsworth S. E., "Motivating children to learn effectively: Exploring the value of instrinsic integration in educational game", Journal of the Learning Science, [www.tandforline.com/loi/htns20], (2011).

[12] Davis F. D., "Perceived usefulness, perceived ease of use, and user acceptance of information technology", MISQuarterly, vol. 13, no. 3, (1989), pp. 319-340.

[13] Igbaria M., "An Examination of the factors contributing to Micro Computer technology acceptance", Journal of Information system, Elsiever Science, USA, (1994).

[14] Adams D. A., Nelson R. R. and Todd P. A., "Perceived usefulness, ease of use, and usage of information technology: A replication", MIS Quarterly, vol. 16, (1992), pp. 227-247.

[15] C. W. Wynne and T. Peter, "On The use Usefulness, ease of use of structural equation Modeling in MIS Research: A note of Caution”, Management Information System Quarterly, vol. 21, no. 3, (1991).

[16] Fishbein M. and Ajzen I., "Belief, attitude, intention, and behavior: An introduction to theory and research", Reading, MA: Addison-Wesley, (1975).

[17] Y. P. Sung, "An Analysis of the Technology Acceptance Model in Understanding University Students' Behavioral Intention to Use e-Learning", Department of Educational Technology, Konkuk University, Seoul, South Korea, (2009).

[18] Hong W., Tong J., Wong W. and Tam K., "Determinants of User Acceptance of Digital Libraries: An Empirical Examination of Individual Differences and System Characteristics", Journal of Management Information Systems / Winter 2001-2002, M.E. Sharpe, Inc., vol. 18, no. 3, (2002), pp. 97-124.

[19] C. C. Min., M. H. Hsu and Eric T.G., "Understanding knowledge sharing in virtual communities: An Integration of social capital and social cognitive theories", Decision Support Systems, science direct, Elsevier, vol. 42, (2006), pp. 1872-1888.

\section{Author}

Munir, is currently a Professor of the Study Program of Computer Science, Faculty of Mathematics and Sciences Education, Indonesia University of Education. He received a $\mathrm{Ph} . \mathrm{D}$ degree in Information Science from Universiti Kebangsaan Malaysia in 2001 on "Multimedia in Education for Literacy". Prof. Munir's research interests are in Multimedia for Education and e-learning. He was Director of ICT Directorate, Indonesia University of Education, Bandung, Indonesia. 